and a control group of Manchester severe asthma patients requiring less than 3 courses of steroids per year. Using case files we collected data including DXA scan results, SCS use and osteoporosis risk factors.

Results BMD change and BTM did not correlate overall (patients $\mathrm{n}=78$, controls $\mathrm{n}=18$ ) but correlated negatively in patients where the most recent DXA was

Conclusion This study has confirmed that BTM may be potentially used as a surrogate for BMD measurement; however a more a prospective study where BTM are measured at the time of two DXA scans which are years apart is required.

\section{P237 A REVIEW OF THE STEROID SPARING IMPACT OF MYCOPHENOLATE MOFETIL IN THE SEVERE ASTHMA POPULATION AT THE NORTH WEST LUNG CENTRE, UNIVERSITY HOSPITAL SOUTH MANCHESTER}

${ }^{1} \mathrm{KE}$ George, ${ }^{1} \mathrm{HGT}$ Brice, ${ }^{1} \mathrm{SJ}$ Fowler, ${ }^{2} \mathrm{U}$ Holmes, ${ }^{2} \mathrm{R}$ Daly, ${ }^{2} \mathrm{RM}$ Niven. ${ }^{1}$ University of Manchester, Manchester, Greater Manchester; ${ }^{2}$ University Hospital South Manchester, Manchester, Greater Manchester

\subsection{6/thoraxjn-2014-206260.365}

Objectives To determine whether Mycophenolate mofetil (MMF) is an effective steroid sparing agent in a single centre cohort of severe asthma patients.

Background MMF is a powerful inhibitor of purine and pyrimidine synthesis via the de novo pathway, upon which lymphocyte production is dependent. It is currently licensed for use in transplant rejection prophylaxis. Other immunosuppressant therapies have been used off licence in difficult-to-treat asthma patients under specialist supervision in an effort to reduce corticosteroid use. MMF is currently a third line immunosuppressant after methotrexate and azathioprine at the North West Lung Centre. Methods A retrospective data analysis was performed including all patients under specialist asthma care at UHSM that were previously or currently treated with MMF. Annualised average daily steroid dose was calculated from the available data in patient case notes. This was calculated for 12 months prior to

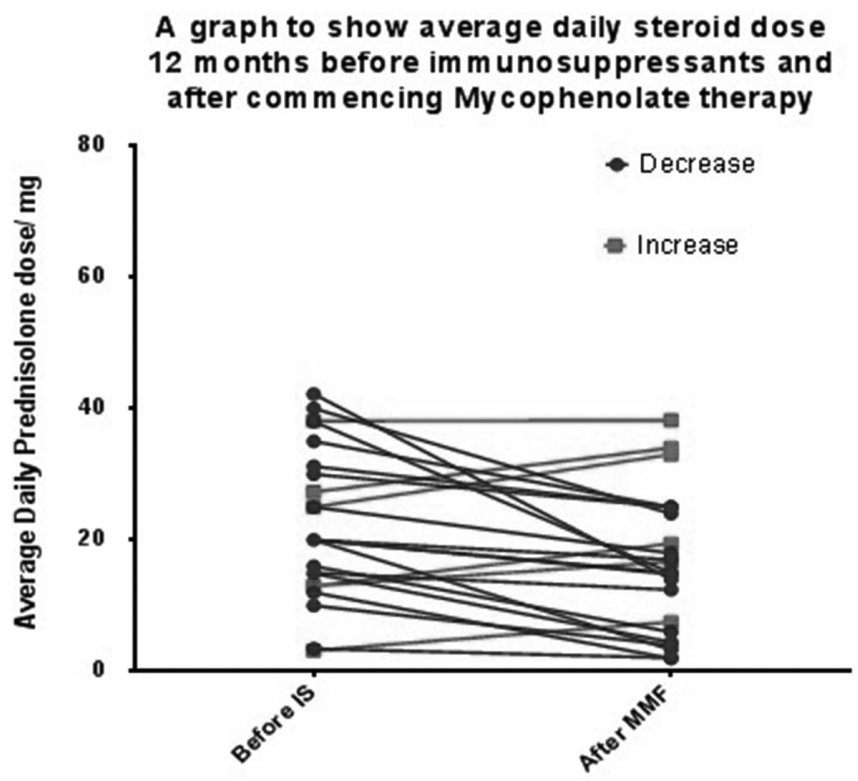

Abstract P237 Figure 1 commencing any immunosuppressant therapy and during MMF treatment. Exacerbation and hospital admission rates were also recorded.

Results A total of 34 patients were identified as being on MMF for severe asthma for at least 8 weeks. 11 did not tolerate MMF or had no response and subsequently stopped. The primary analysis was carried out on 23 patients and a secondary post hoc analysis was performed on all patients who had been on treatment for a minimumof 6 months at the time of the study $(\mathrm{N}=12)$. The average yearly steroid sparing impact of MMF was $5.9 \mathrm{mg}$ per day, $(\mathrm{p}<0.005) .74 \%$ had an overall reduction and $35 \%$ achieved a reduction of $10 \mathrm{mg}$ or more. This value was lower in those who had been on treatment for $>6$ months $(\Delta$ $3.9 \mathrm{p}=0.20$ ). There was no statistically significant reduction in admission or exacerbation rates.

Conclusion MMF has shown a small steroid sparing effect in this retrospective analysis, although the effect appeared less positive in the sub-group of those analysed after being on treatment for at least 6 months further analysis of the potential benefits of MMF in this patient population is required.

\section{P238 THE EFFECT OF INHALATION DURATION ON LUNG DEPOSITION WITH A PRESSURISED METERED-DOSE INHALER (PMDI)}

${ }^{1} \mathrm{C}$ Van Holsbeke, ${ }^{2} \mathrm{~J}$ Marshall, ${ }^{1} \mathrm{~J}$ De Backer, 'W Vos. ${ }^{1}$ Fluidda Nv, Kontich, Belgium; ${ }^{2}$ Mundipharma International, Cambridge, UK

10.1136/thoraxjnl-2014-206260.366

Rationale Although the guidance for using a pMDI is to inhale 'slow and deeply', many patients inhale fast over a short duration. The ERS/ISAM Task Force suggested 'slowly' equates to inhaling over 4-5 seconds (s) for adults1, a much clearer instruction. This study therefore examined the influence of inhalation time on total lung deposition (TLD) using Functional Respiratory Imaging (FRI).

Methods Three-dimensional airway models of 6 asthma patients (mean FEV1 83\%), treated with an ICS/LABA combination, were included. The lung deposition characteristics of an HFAbased pMDI (MMAD $\sim 3.0 \mu \mathrm{m}$; fine particle fraction (FPF) $\sim 40 \%$ ) were assessed using FRI. Simulations were performed on 3 different inhalation profiles matched for the same inspiratory volume $(3 \mathrm{~L})$ with durations of $1 \mathrm{~s}, 3 \mathrm{~s}$ and $5 \mathrm{~s}$ and actuation at start of inhalation.

Results For the $1 \mathrm{~s}, 3 \mathrm{~s}$ and $5 \mathrm{~s}$ profiles, the TLD values were $22.81 \pm 3.71 \%, 36.13 \pm 2.51 \%$ and $41.61 \pm 3.11 \%$ of nominal dose respectively, and were predicted using a concave down quadratic model $(\mathrm{R} 2=0.87, \mathrm{p}<0.001)$. The central to peripheral deposition ratios were $1.58,0.81$ and 0.57 respectively.

Conclusions A $5 \mathrm{~s}$ inhalation led to highest TLD with greatest peripheral deposition. Increased deposition with longer times mainly reflected increased peripheral deposition, central deposition was less affected by flow rate. These data support ERS/ ISAM guidance for inhaling over 4-5 sec to optimise deposition, although similar TLD were achieved with $3 \mathrm{~s}$. These data also suggest that high FPF pMDIs can achieve reasonable deposition even with short, fast inhalations.

\section{REFERENCES}

1 Laube BL, et al. ERJ 2011;37(6):1308-417

Disclaimer acknowledgement: Reproduced with permission of the European Respiratory Society: Eur Resp J September 2014; 44, Suppl. 58: In Press 